\title{
An Efficient Electric Charge Transfer Device for Intelligent Storage Units
}

\author{
Ahmed Rebbani' ${ }^{1}$, Omar Bouattane ${ }^{1}$, Lhoucine Bahatti', Mimoun Zazoui ${ }^{2}$ \\ ${ }^{1}$ Lab SSDIA, ENSET, Hassan II University, Mohammedia, Morocco \\ ${ }^{2}$ Lab PMCER, FST, Hassan II University, Mohammedia, Morocco \\ Email: a.rebbani@gmail.com
}

Received 25 July 2014; revised 21 August 2014; accepted 31 August 2014

Copyright (C) 2014 by authors and Scientific Research Publishing Inc.

This work is licensed under the Creative Commons Attribution International License (CC BY). http://creativecommons.org/licenses/by/4.0/

c) (i) Open Access

\begin{abstract}
This paper deals with a dynamic analysis of an optimal technique used for direct electrical energy storage, where a concept of charge transfer between different electric storage units is used. This analysis is developed to seek for efficient and real time conditions to maintain optimal behavior for charge recovery from intermittent power sources in the field of renewable energies like solar and wind. The proposed analysis leads to elaborating a set of interesting states and conditions that allows the user to choose effective configuration parameters that lead to an optimal or near optimal charge transfer device. The proposed device is designed to ensure an optimal transfer of electric charges. It must be self-configured to retrieve and transfer the maximum energy from the sources to the storage units (Super-capacitors, batteries...). Some interesting results, by simulating the proposed device, are presented to show how this optimization problem can be viewed as a combinatorial one, where the optimization algorithm is asked to find the suitable switching combination to configure the device to be closest to the optimal charge recovery.
\end{abstract}

\section{Keywords}

Energy Storage, Charge Transfer, Renewable Energy, Photovoltaic, Supercapacitor, Switching

\section{Introduction}

Autonomy and energy independence are considered as the major contemporary challenges for humanity. However, such autonomy requires solving the problem of energy storage, especially in remote locations where the use of renewable energy is increasingly dominant [1]-[3]. The most used storage systems in the smallest scale, stationary or embedded, are based on batteries that allow long-term storage. However, this storage medium is not very convenient for low energy level and high power demand applications (for example when starting en- 
gines), [4]. Indeed, the SCs are the electrical power components able to store energy directly as electric charges; this is completely different from the battery storage process that is based on a chemical reaction to store electricity [5] [6]. In the SC, the electrical energy is stored or released much faster since there is no electrochemical process [7]. A SC can be charged and discharged faster than the conventional batteries. Also it can provide an extremely high power during a short slot time [8].

Super Capacitor technology is a new process that has achieved the capacity of several thousand Farads, with high specific power. This technology demonstrates its usefulness and suitability for future smart grids, especially when coupled with Lithium-Ion batteries (LIC: Lithium Ion Capacitor) [9] [10].

In the near future, the SC of high energy density will certainly be the most efficient way to store electricity supplied by renewable sources [11] [12]. Subsequently, it should imply a massive development of photovoltaic devices. It is interesting to notice that, the increasing rate of the switched power and the reduced cost of power semiconductor components have easily enabled the development of new topologies for power electronic applications, in which various switches are perfectly controlled to direct high energy [13].

Another configuration, commonly used by modern storage systems and dedicated to remote sites, is based on a (battery, SC) hybrid storage system. Batteries are used to meet energy needs while SCs are used to satisfy the significant peak demand for power [4] [14]-[17].

In this paper, we present the design and simulation of an electrical charge transfer system assigned to the storage of energy application. It is specially designed using SC and controlled switches.

In the second section we discuss the electric charge transfer from a renewable source (PV, wind...) to a storage unit using an Individual Capacitor Switching Process (ICSP). Section III examines the charge transfer using another technique named cumulative capacitor switching process (CCSP). Section IV gives a detailed study of the switching technique called Global Parallel Switching Process (GPSP) which is proposed as the third process for energy storage. Section V presents a comparative study in terms of two principals' aspects that are: energy, dynamic behavior and efficiency ratio, between ICSP and CCSP techniques, since GPSP will be viewed as a particular case of CCSP. In Section VI, the device circuit simulation and dynamic behavior of the storage unit (C) are presented according to the different proposed techniques. The final section is devoted to sum concluding remarks and future perspectives.

\section{Individual Capacitor Switching Process (ICSP)}

The basic diagrams of Figure 1 show the principle of energy transfer from a renewable source $(E)$ to a storage unit $(C)$ through a storage tank essentially composed of a set of branches. Each branch consists of a controlled switches $\left(\mathrm{K}_{i}\right)$ associated to a subset of SC leading to an equivalent capacitor $\left(C_{i}\right)$, as in Figure 1(a).

The equivalent SC used in each branch (cell) is considered as an ideal capacitance $C_{i}$, connected in series with an internal equivalent resistor $R_{i}$ [18] [19]. The storage tank is equivalent to a polymorphic capacitor consisting of the elementary SC connected using different series and parallel configurations.

The circuit of Figure 1(a) is characterized by:

- E: Renewable energy source,

- $C$ : Storage Unit,

- $\left\{C_{i}, \mathrm{~K}_{i}\right\}=\left(\mathrm{Br}_{i}\right)$ : Capacitor branch numbered $i$.

At the initial time $t=t_{0}$, we start from the following initial conditions: $\mathrm{K}_{0}$ : “OFF", $\mathrm{K}_{0}^{\prime}$ : “ON" and all $\mathrm{K}_{i \text { (i=1 to }}$ n): "ON" so that, all capacitors $C_{i}$ will be loaded to $E\left(V c_{i}(0)=E\right)$.

At time $t=t_{1}$, the renewable source $E$ is isolated from the container ( $\mathrm{K}_{0}^{\prime}:$ "OFF”, $\mathrm{K}_{i}(i=1$ to $n)$ : “OFF"), and then $\mathrm{K}_{0}$ and $\mathrm{K}_{1}$ are closed, so that $C_{1}$ is switched to $C$ (Figure 2(b)).

At the steady state, the voltage $V^{\prime}$ of the circuit is, as in [20]:

$$
V^{\prime}=V^{(1)}=\frac{V^{(0)} \cdot C+E \cdot C_{1}}{C+C_{1}}
$$

At time $t=t_{2}$, we open $\mathrm{K}_{1}$ and close $\mathrm{K}_{2}\left(\mathrm{~K}_{1}\right.$ : “OFF" and $\mathrm{K}_{2}$ : "ON"), therefore $C_{2}$ will be connected to $C$, and the second steady state voltage is:

$$
V^{(2)}=\frac{V^{(1)} \cdot C+E \cdot C_{2}}{C+C_{2}}
$$


(Polymorphic Capacitor)

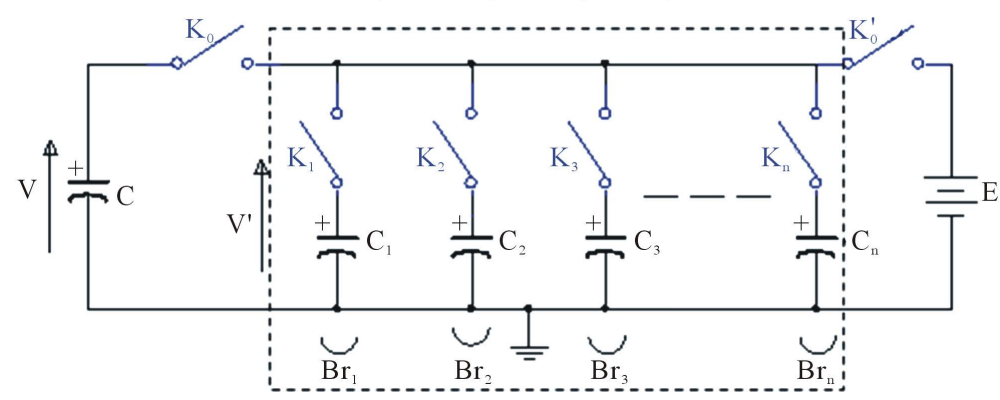

(a)

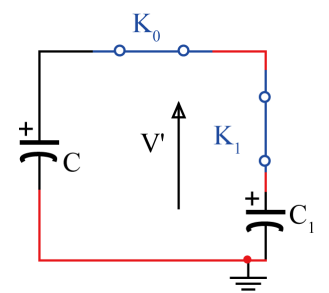

(b)

Figure 1. Charge transfer by polymorphic capacitor system. (a) Basic diagram of charge transfer from $E$ to $C_{i}$; (b) Basic circuit of charge transfer from $C_{1}$ to $C$.

At time $t=t_{n}, \mathrm{~K}_{n-1}$ is switched $\mathrm{OFF}$ and $\mathrm{K}_{n} \mathrm{ON}$, so, $C_{n}$ is connected to $C$ then:

$$
V^{(n)}=\frac{V^{(n-1)} \cdot C+E \cdot C_{n}}{C+C_{n}}
$$

Combining the above recurrent equations, we have:

$$
V^{(n)}=\frac{C^{2} V^{(n-2)}}{\left(C+C_{n}\right) \cdot\left(C+C_{n-1}\right)}+E \cdot\left(\frac{C \cdot C_{n-1}}{\left(C+C_{n}\right) \cdot\left(C+C_{n-1}\right)}+\frac{C_{n}}{C+C_{n}}\right)
$$

The generalized equation of $V^{(n)}$ is:

$$
V^{(n)}=\frac{C^{n}}{\prod_{i=1}^{n}\left(C+C_{i}\right)} \cdot V^{(0)}+E \cdot \sum_{i=0}^{n-1}\left(\frac{C^{i} \cdot C_{n-i}}{\prod_{j=0}^{i}\left(C+C_{n-j}\right)}\right)
$$

Equation (2) governs the general operation of the system whatever $C_{i}$, but in practice and to ensure optimal energy transfer from $C_{i}$ to $C$, we must take $C_{i}=\alpha \cdot C$ and $\alpha=0.75$ [21].

In a simplified case, we assume that $C_{i}=C_{1}$ whatever $i$, so,

$$
V^{(n)}=\frac{V^{(n-1)} \cdot C+E \cdot C_{1}}{C+C_{1}} \text { and } V^{(n-1)}=\frac{V^{(n-2)} \cdot C+E \cdot C_{1}}{C+C_{1}}
$$

Then,

$$
V^{(n)}=V^{(n-2)} \cdot\left(\frac{C}{C+C_{1}}\right)^{2}+E \cdot \frac{C_{1}}{C+C_{1}}(1+C)
$$

Generalizing Equation (3), we obtain for $n \geq 1$;

$$
V^{(n)}=V^{(0)} \cdot\left(\frac{C}{C+C_{1}}\right)^{n}+E \cdot \frac{C_{1}}{C+C_{1}}\left[\sum_{i=0}^{n-1}\left(\frac{C}{C+C_{1}}\right)^{i}\right] \quad(n \geq 1)
$$


Taking $\frac{C}{C+C_{1}}=a<1$, Since;

$$
\sum_{i=0}^{n-1}\left(\frac{C}{C+C_{1}}\right)^{i}=\sum_{i=0}^{n-1}(a)^{i}=\frac{1-a^{n}}{1-a}
$$

Then;

$$
V^{(n)}=V^{(0)} \cdot a^{n}+E \cdot \frac{C_{1}}{C+C_{1}}\left[\frac{1-a^{n}}{1-a}\right]=V^{(0)} \cdot a^{n}+E \cdot\left(1-a^{n}\right)
$$

In each switching phase $n$, the constant time which governs the transfer of charge between each $C_{n}=C_{1}$ and $C$ is:

$$
\tau_{n}=\left(R+R_{1}\right) \cdot \frac{C \cdot C_{1}}{C+C_{1}}
$$

where: $R$ is the internal equivalent serial resistor of $C$ and $R_{1}$ internal equivalent serial resistor of $C_{1}$.

The stored energy in $C$ is:

$$
W_{c}(n)=\frac{1}{2} \cdot C \cdot\left(\left(V^{(0)}-E\right) \cdot a^{n}+E\right)^{2}
$$

For a maximum energy transfer from $C_{1}$ to $C$, we take $C_{1}=\alpha \cdot C$ where $\alpha=0.7$ [20] [21].

So, $a=1 /(1+\alpha)=4 / 7=0.57$, then;

$$
W_{c}(n)=\frac{1}{2} \cdot C \cdot\left(\left(V^{(0)}-E\right) \cdot\left(\frac{1}{1+}\right)^{n}+E\right)^{2}
$$

And,

$$
\tau_{n}=\left(R+R_{1}\right) \cdot \frac{\alpha \cdot C}{1+\alpha}
$$

For an arbitrary numerical example where: $C=2 \mathrm{~F}, C_{1}=1.5 \mathrm{~F}, V^{(0)}=6 \mathrm{~V}$ and $E=12 \mathrm{~V}$, the stored energy in $C$ depends on $n$ as presented Figure 2.

Also, in Figure 2, we can see that the variation of the stored energy becomes non significant for $n>5$. We can conclude that after 5 switching iterations, the storage unit will receive almost its complete load.

\section{Cumulative Capacitor Switching Process (CCSP)}

\subsection{Simple Cumulative Capacitor Switching Process (SCCSP)}

The simple cumulative switching device is a successively branches switching model. At each step $i$, the branch

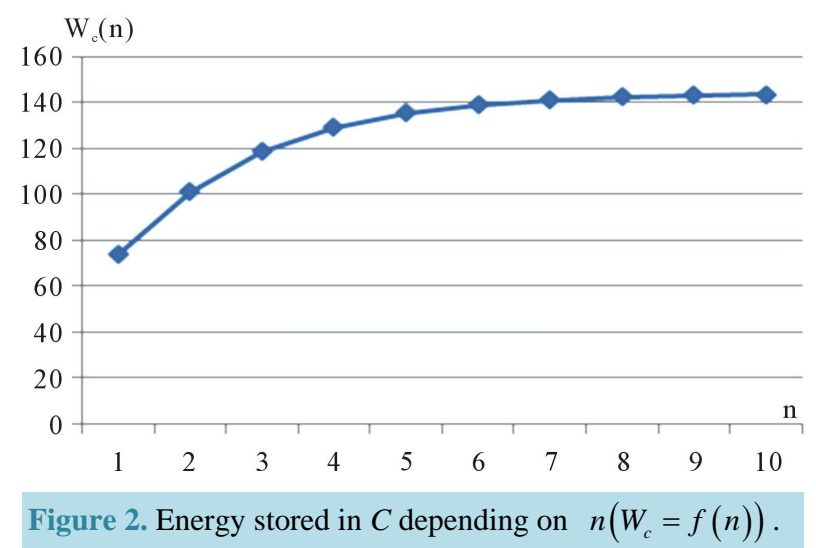


$\mathrm{Br}_{i}$, is connected to the storage capacitor $C$ by closing its corresponding switch $\mathrm{K}_{i}$ and letting the previous switches in their closed state (see Figure 3).

The functional sequence of this device is as follows:

- Initial settings: $\mathrm{K}_{0}$ is "OFF", the $\mathrm{K}_{i(i=1 \text { to } n)}$ are "ON", and $\mathrm{K}_{0}^{\prime}$ is "ON", all the capacitors $C_{i}$ are loaded to $E$.

- Starting step: At $t=t_{1}, \mathrm{~K}_{0}^{\prime}$ and all $\mathrm{K}_{i(i=1 \text { to n) }}$ are opened (the source $E$ and the capacitors $C_{i(i=1 \text { to } n)}$ are disconnected from the circuit), then we start by closing $\mathrm{K}_{0}$ and $\mathrm{K}_{1}$

At this first step, the steady state voltage of the circuit is:

$$
V^{(1)}=\frac{V^{(0)} \cdot C+E \cdot C_{1}}{C+C_{1}}
$$

- At $\left(t=t_{2}\right), \mathrm{K}_{2}$ is closed and $\mathrm{K}_{1}$ remains so, $\left(\mathrm{K}_{1}\right.$ and $\mathrm{K}_{2}$ are "ON"), the steady state voltage of the circuit becomes:

$$
V^{(2)}=\frac{V^{(1)} \cdot C_{e q 1}+E \cdot C_{2}}{C_{e q 1}+C_{2}}
$$

where $C_{e q 1}=C / / C_{1}=C+C_{1}$

$$
V^{(2)}=\frac{V^{(0)} \cdot C+E \cdot\left(C_{1}+C_{2}\right)}{C+C_{1}+C_{2}}
$$

- At $\left(t=t_{3}\right), \mathrm{K}_{3}$ is closed and $\mathrm{K}_{1}$ and $\mathrm{K}_{2}$ remain so, $\left(\mathrm{K}_{1}, \mathrm{~K}_{2}\right.$ and $\mathrm{K}_{3}$ are "ON"), the steady state voltage of the circuit is:

$$
V^{(3)}=\frac{V^{(2)} \cdot C_{e q 2}+E \cdot C_{3}}{C_{e q 2}+C_{3}}
$$

where: $C_{e q 2}=C / / C_{1} / / C_{2}=C+C_{1}+C_{2}$ then;

$$
V^{(3)}=\frac{V^{(0)} \cdot C+E \cdot\left(C_{1}+C_{2}+C_{3}\right)}{C+C_{1}+C_{2}+C_{3}}
$$

Generally at the stage $t=t_{n}$, we have:

$$
V^{(n)}=\frac{V^{(0)} \cdot C+E \cdot \sum_{i=1}^{n} C_{i}}{C+\sum_{i=1}^{n} C_{i}}
$$

In the following assumptions as in [20] [21] where: $\forall i, C_{i}=C_{1}=\alpha \cdot C$ (where $\alpha=0.75$ ), we obtain:

$$
V^{(n)}=\frac{V^{(0)}+n \cdot \alpha \cdot E}{1+n \cdot \alpha}
$$

At each step $i$, the used storage unit (C) is charged according to the constant time $\tau_{i(i=1 \text { to } n)}$, that depends on the $\mathrm{K}_{i}$ switches states.

For $\mathrm{K}_{1}$ : "ON" and $\mathrm{K}_{i(i=2 \text { to })}$ : “OFF"; $\tau_{1}=\left(R+R_{1}\right) \frac{C \cdot C_{1}}{C+C_{1}}$

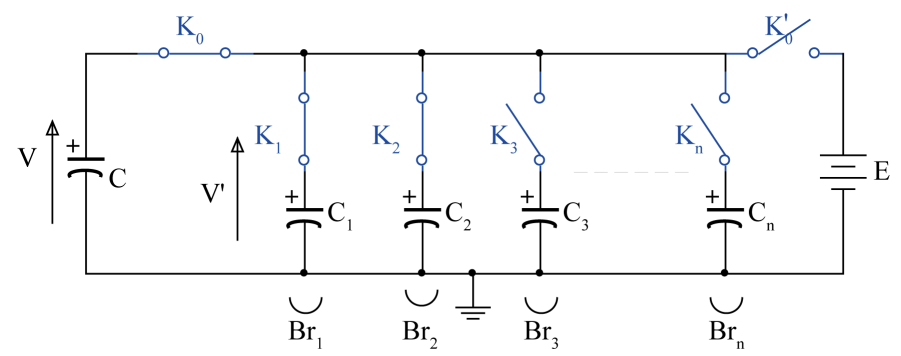

Figure 3. Simple cumulative capacitor switching process (SCCSP). 
For $\mathrm{K}_{1,2}$ "ON" and $\mathrm{K}_{i(i=3 \text { to } n)}$ "OFF"; we have: $R_{e q}=\left(R / / R_{1}\right)+R$ and $C_{e q}=\left(C+C_{1}\right) \cdot C_{2} / C+C_{1}+C_{2}$ (i.e. $\left(C / / C_{1}\right)$ serial connected to $\left.C_{2}\right)$

So,

$$
\tau_{2}=\frac{\left(R \cdot R_{1}+R \cdot R_{2}+R_{1} R_{2}\right)}{\left(R+R_{1}\right)} \cdot \frac{\left(C+C_{1}\right) \cdot C_{2}}{\left(C+C_{1}+C_{2}\right)}
$$

At the general step $n$, the circuit is viewed as:

$$
R_{e q_{n}}=\left(R / / R_{1} / / R_{2} / / \cdots / / R_{n-1}\right)+R_{n}=\frac{1}{\sum_{i=1}^{n-1} \frac{1}{R_{i}}+\frac{1}{R}}+R_{n} \text { and } C_{e q_{n}}=\frac{C_{n} \cdot\left(C+\sum_{i=1}^{n-1} C_{i}\right)}{C+\sum_{i=1}^{n} C_{i}}
$$

This leads to: $\tau_{n}=\left(\frac{1}{\sum_{i=1}^{n-1} \frac{1}{R_{i}}+\frac{1}{R}}+R_{n}\right) \cdot\left(\frac{C_{n} \cdot\left(C+\sum_{i=1}^{n-1} C_{i}\right)}{C+\sum_{i=1}^{n} C_{i}}\right)$

If we consider that all the capacitors are same, this is the simplified context where: $\forall i, R_{i}=R_{1}$ and $C_{i}=C_{1}$ then $R_{e q_{n}}=R_{1}+\frac{R_{1} \cdot R}{R_{1}+(n-1) \cdot R}$ and $C_{e q_{n}}=\frac{(n-1) C_{1}^{2}+C \cdot C_{1}}{C+n C_{1}}$

So,

$$
\tau_{n}=\left(R_{1}+\frac{R_{1} \cdot R}{R_{1}+(n-1) \cdot R}\right) \cdot\left(\frac{(n-1) C_{1}^{2}+C \cdot C_{1}}{C+n C_{1}}\right)
$$

The stored energy in $C$ is:

$$
W_{c}(n)=\frac{1}{2} \cdot C \cdot\left(\frac{V^{(0)}+n \cdot \alpha \cdot E}{1+n \cdot \alpha}\right)^{2}
$$

Figure 4 shows the curve of the stored energy in $(C)$ according to the cumulative capacitor switching process. In Figure 4, we can see that, it is necessary to switch $\mathrm{ON}$ at least 10 capacitors to transfer a maximal energy to $C$.

Due to the successive branches connecting to the storage unit $C$, the equivalent capacitor of the circuit is modified at each switching step. Also, we can see that, a part of the energy of the next branch is consumed by the branches already switched; this corresponds to a weakness of this method.

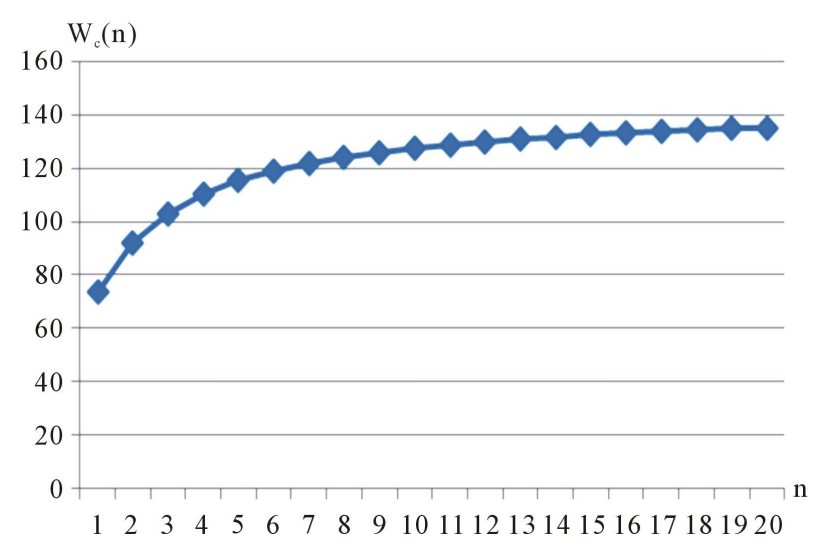

Figure 4. Energy stored in $(C)$ versus the number of cumulative switched Capacitor, $W_{c}(n)=f(n)$. 


\subsection{Optimal Cumulative Capacitor Switching Process (OCCSP)}

To optimize energy transfer during each switching step, it is required that $C_{i}=\alpha \cdot C_{e q(i-1)}$ [21]. In this case, we must satisfy the following settings:

$$
\begin{gathered}
C_{1}=\alpha \cdot C, \\
C_{e q 1}=C / / C_{1}=C+C_{1}=(1+\alpha) \cdot C, \\
C_{e q 2}=C_{e q 1} / / C_{2}=(1+\alpha)^{2} \cdot C
\end{gathered}
$$

So, $C_{e q i}=(1+\alpha)^{i} \cdot C$ and $C_{i}=\alpha \cdot C_{e q(i-1)}=\alpha \cdot(1+\alpha)^{i-1} \cdot C$, for $i \geq 1$

According to Equation (9) we find:

$$
V^{(n)}=\frac{V^{(0)}-E}{\sum_{i=1}^{n}(1+\alpha)^{i}}+E
$$

Therefore the energy stored in the storage unit at the state $n$ is:

$$
W_{c}^{(n)}=\frac{1}{2} \cdot C \cdot\left(\frac{V^{(0)}-E}{\sum_{i=1}^{n}(1+\alpha)^{i}}+E\right)^{2}
$$

In the following graphs of Figure 5(a), we show a comparison of the amounts of accumulated energy in the storage unit according to the SCCSP and OCCSP. While in Figure 5(b), we show the energetic difference between the two techniques using the following settings: $V(0)=6 \mathrm{~V}, C=2 \mathrm{~F}, E=12 \mathrm{~V}$ and $\alpha=0.75$.

From these figure we see that, the optimal difference is obtained for $n=3$ capacitor branches.

\section{Global Parallel Switching Process (GPSP)}

\subsection{Simple Global Parallel Switching Process (SGPSP)}

In this part, we consider that, all the $C_{i}$ capacitors are the same $\left(C_{i}=C_{1}\right)$ and they are loaded to $E$. Then, the charged circuit is connected to $C$. The steady state voltage of the resulted circuit is:

$$
V^{\prime}=\frac{V^{(0)} \cdot C+n \cdot C_{1} \cdot E}{C+n \cdot C_{1}}
$$

If $C_{1}=\alpha \cdot C$ then

$$
V^{\prime}=\frac{V^{(0)}+n \cdot \alpha \cdot E}{1+n \cdot \alpha}
$$

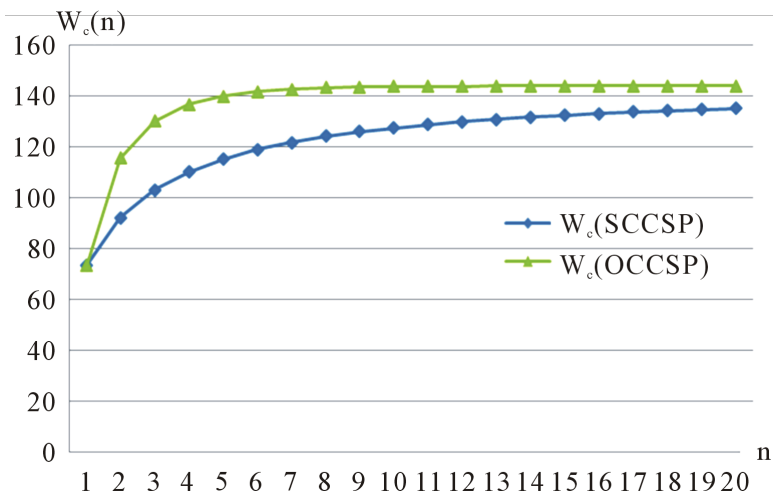

(a)

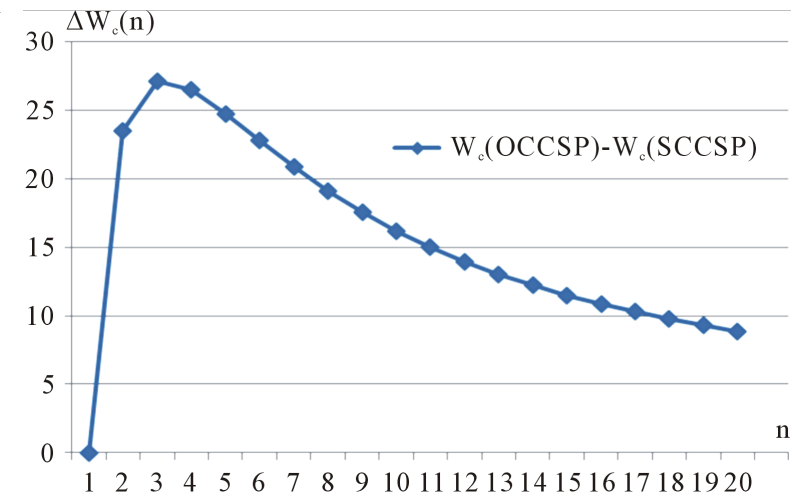

(b)

Figure 5. Energy assessment between OCCSP and SCCSP processes. (a) Energy stored in $C$ versus $n$ for SCCSP and OCCSP, $W_{c}=f(n)$; (b) Energy difference between OCCSP and SCCSP $\Delta W_{c}=f(n)$. 
$V^{\prime}$ corresponds to the same equation $V^{(n)}$ of (10), indeed this process is the same as a simple cumulative capacitor switching process (SCCSP) corresponding to zero switching slot times $\left(t_{1}=t_{2}=\cdots=t_{n}\right)$.

Figure 6(a), indicates the amount of energy stored in $C$ for the case of the same initial conditions as in the previous sections, $\left(V^{(0)}=6 \mathrm{~V}, C=2 \mathrm{~F}, E=12 \mathrm{~V}\right.$ and $\left.\alpha=0.75\right)$.

We can conclude from this study that, the Global Parallel Switching Process remains a particular case of the simple cumulative capacitor switching process.

\subsection{Optimal Global Parallel Switching (OGPSP)}

The simple global parallel switching process (SGPSP) can be improved by choosing the values of $C_{i}$ of the same range as those selected in the optimal cumulative capacitor switching process.

$$
C_{1}=\alpha \cdot C, C_{2}=\alpha \cdot\left(C+C_{1}\right), C_{3}=\alpha\left(C+C_{1}+C_{2}\right) \text {, and } C_{4}=\alpha\left(C+C_{1}+C_{2}+C_{3}\right) \text { etc. }
$$

In the global switching case ( $\mathrm{K}_{i(1 \text { to } n)}$ are "ON"), the equivalent capacitance which will be connected to $C$ is:

$$
C_{e q_{n}}=\sum_{i=1}^{n} C_{i}=\alpha \cdot\left[n \cdot C+\sum_{i=1}^{n}\left((n-i) \cdot C_{i}\right)\right]
$$

The final steady state voltage across $C$ is:

$$
V^{(n)}=\frac{V^{(0)} \cdot C+E \cdot \alpha \cdot\left[n \cdot C+\sum_{i=1}^{n}\left((n-i) \cdot C_{i}\right)\right]}{C+\alpha \cdot\left[n \cdot C+\sum_{i=1}^{n}\left((n-i) \cdot C_{i}\right)\right]}
$$

And the total energy stored in $C$ is:

$$
W_{c}^{(n)}=\frac{1}{2} \cdot C\left(\left(\frac{V^{(0)} \cdot C+E \cdot \alpha \cdot\left[n \cdot C+\sum_{i=1}^{n}\left((n-i) \cdot C_{i}\right)\right]}{C+\alpha \cdot\left[n \cdot C+\sum_{i=1}^{n}\left((n-i) \cdot C_{i}\right)\right]}\right)^{2}\right)
$$

The Figure 6(b) compares the energy storage process in $(C)$ according to both techniques of simple and optimal global parallel switching processes.

\section{Comparative Analysis of Energy Storage between ICSP and SCCSP}

\subsection{Energy and Dynamic Aspects}

The following figure shows the values of the energy stored in $C(2 \mathrm{~F})$ and differences of the stored energy between two successive switching steps $(n-1$ and $n)$ :

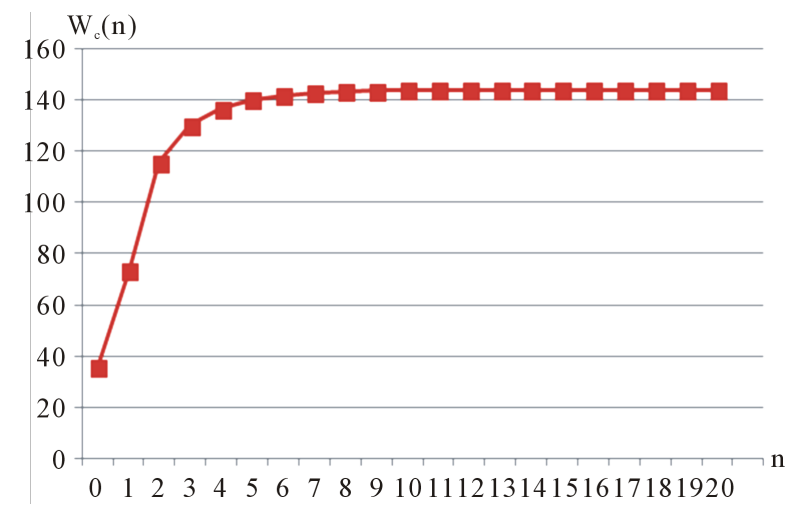

(a)

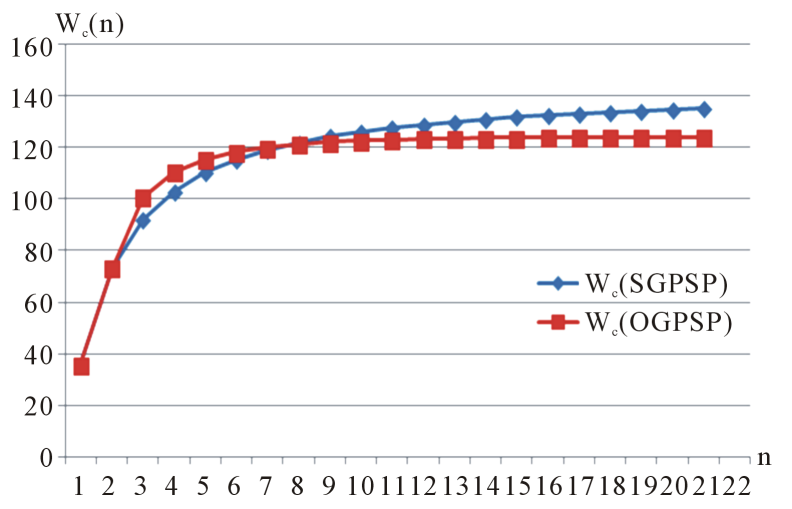

(b)

Figure 6. Energy stored in $C$ for SGPSP and OGPSP $\left[W_{c}=f(n)\right]$. (a) Energy stored in $C$ for SGPSP $\left[W_{c}=f(n)\right]$; (b) Energy stored in $(C)$ versus number of SC for SGPSP and OGPSP $\left(W_{c}=f(n)\right)$. 
In Figure 7(a), we present the evolution of the variation of the stored energy in $C$ as a function of $n$. The ICSP has a speed greater than the SCCSP for a reduced number of capacitors. Beyond five capacitors the variations are minimal. From the Figure 7(b), which compares the dynamic aspect of the two switching processes ICSP and SCCSP, we conclude that the individual switching is faster and more economical than the cumulative one.

In conclusion, the optimal solution for maximum energy transfer with minimal number of capacitors is the Individual Capacitor Switching Process.

\subsection{Energy Lost during the Transition from One State to Another in the ICSP}

In order to assess the percentage of energy losses in this system, the ratio $\frac{W_{i}-W_{f}}{W_{i}}=1-\frac{W_{f}}{W_{i}}$ is calculated, where,

$W_{i}$ (initial energy) is the sum of the energy stored in $C$ at the step $(n-1)$ and the one stored in $C_{n}$, where $C_{n}$ is the next capacitor to be connected to $C$.

$W_{f}$ (final energy) is the sum of the energy stored in $C$ and $C_{n}$ at the step $n$.

At the step $n-1$, the stored energy in $C$ is:

$$
W_{c}^{(n-1)}=\frac{1}{2} \cdot C \cdot\left(\frac{V^{(0)}-E}{(1+\alpha)^{n-1}}+E\right)^{2},
$$

using Equation (7), the energy stored in any capacitor $C_{i}$ is:

where, $C_{i}=C_{1}=\alpha \cdot C$.

$$
W_{C_{i}}^{(n-1)}=\frac{1}{2} C_{1} E^{2}=\frac{1}{2} \cdot \alpha \cdot C \cdot E^{2}
$$

So the total initial energy is:

$$
W_{i}=\frac{1}{2} \cdot C \cdot\left(\frac{V^{(0)}-E}{(1+\alpha)^{n-1}}+E\right)^{2}+\frac{1}{2} \cdot \alpha \cdot C \cdot E^{2}=\frac{1}{2} \cdot C \cdot\left[\left(\frac{V^{(0)}-E}{(1+\alpha)^{n-1}}+E\right)^{2}+\alpha \cdot E^{2}\right]
$$

At the step $n$ :

$$
W_{c}^{(n)}=\frac{1}{2} \cdot C \cdot\left(\frac{V^{(0)}-E}{(1+\alpha)^{n}}+E\right)^{2}
$$

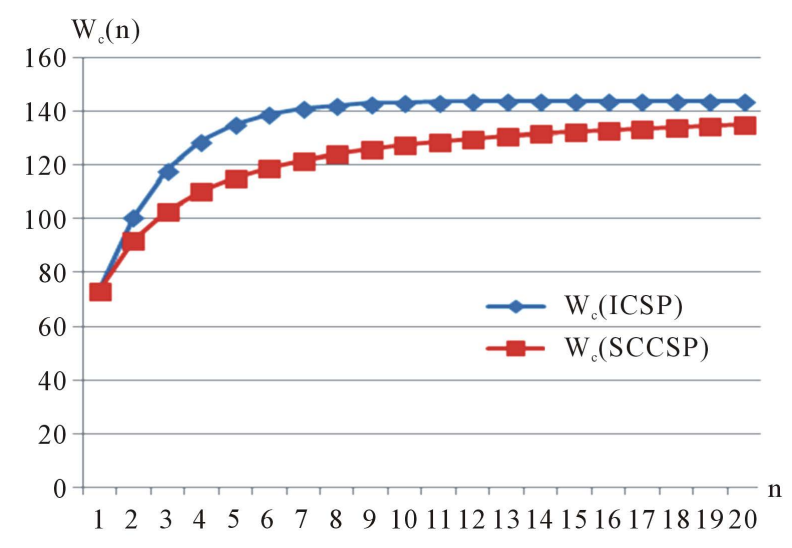

(a)

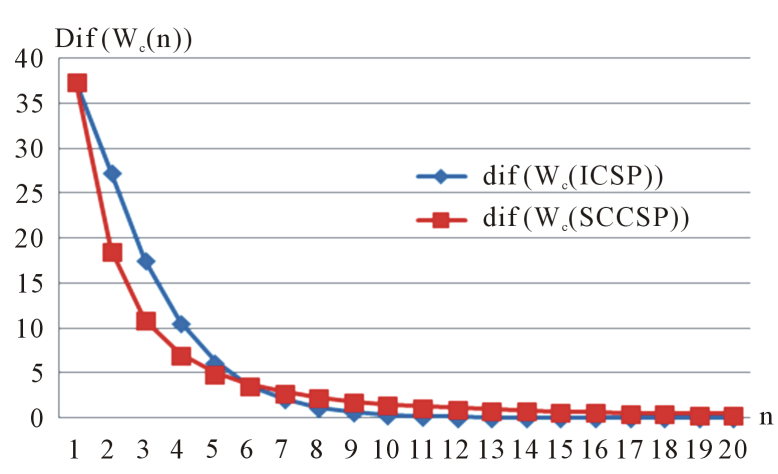

(b)

Figure 7. Energy stored in $C$ and differences between two successive switching steps for ICSP and SCCSP. (a) Energy stored in $C$ versus the number of SC for ICSP and SCCSP; (b) $W_{c}(n)-W_{c}(n-1)=f(n)$ For each Process ICSP and SCCSP. 
And

$$
W_{C_{i}}^{(n)}=\frac{1}{2} \cdot \alpha \cdot C \cdot\left(\frac{V^{(0)}-E}{(1+\alpha)^{n}}+E\right)^{2}
$$

Then the final total energy is:

$$
W_{f}^{(n)}=\frac{1}{2} \cdot C \cdot(1+\alpha) \cdot\left(\frac{V^{(0)}-E}{(1+\alpha)^{n}}+E\right)^{2}
$$

And the ratio:

$$
\frac{W_{f}}{W_{i}}=\frac{(1+\alpha) \cdot\left(\frac{V^{(0)}-E}{(1+\alpha)^{n}}+E\right)^{2}}{\left(\frac{V^{(0)}-E}{(1+\alpha)^{n-1}}+E\right)^{2}+\alpha \cdot E^{2}}
$$

Figure 8 represents the percentage of energy loss during the switching phase from state $(n-1)$ to $(n)$ in the individual parallel switching topology.

As we see in Figure 8, the energy loss ratio tends to 0 after connecting 5 capacitors $(n=5)$, this means that the storage unit $C$ is full of electric charge.

\section{Simulation and Dynamic Behavior of the System}

The diagram of Figure 9 shows the basic simulating circuit designed under National Instrument Multisim simulation platform.

Table 1 shows the code sequences used in the circuit to control the different switches at a given frequency.

The following figures illustrate the variations of the voltages of the storage unit $C$ and the capacitor $C_{1}$ according to the individual capacitor switching process, by activating the set of the $K_{i}$ switches.

For Figure 10(a), all these measures are obtained for a low frequency $(10 \mathrm{~Hz})$ of the sequencer. This frequency is chosen low to show the steady state voltage of $C$ when each capacitor $C_{i}$ is included in the circuit.

For another frequency value of the sequencer $(50 \mathrm{~Hz})$, the switches are fast controlled so that the capacitors remain in their linear behaviors. Their steady states are not reached. Thus, the charge/discharge equations of the different capacitors are approximately represented by their linear parts (see Figure 10(b)).

Figure 11(a) shows a comparison of the dynamic behavior of the charge of $C$ in an ICSP (Red) and a SCCSP (Black).

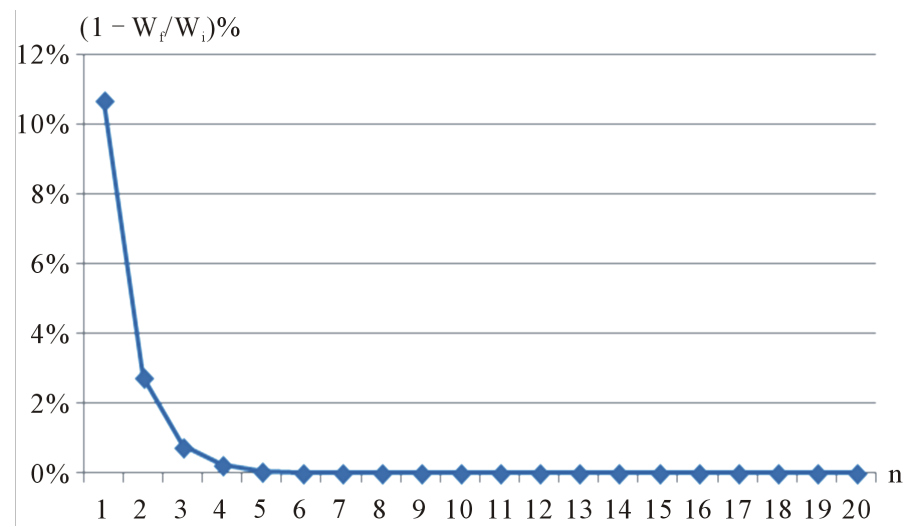

Figure 8. Energy loss during the switching phase from state $(n-1)$ to $(n)$. 
Table 1. Code sequences to control $\mathrm{K}_{i}$.

\begin{tabular}{ccccccccc}
\hline $\mathrm{K}_{0}^{\prime}$ & $\mathrm{K}_{n}$ & $\mathrm{~K}_{5}$ & $\mathrm{~K}_{4}$ & $\mathrm{~K}_{3}$ & $\mathrm{~K}_{2}$ & $\mathrm{~K}_{1}$ & $\mathrm{~K}_{0}$ & Operation \\
\hline 1 & 1 & 1 & 1 & 1 & 1 & 1 & 0 & loading $C_{i}$ from $E$ \\
0 & 0 & 0 & 0 & 0 & 0 & 0 & 1 & $C$ is connected to the DC bus \\
0 & 0 & 0 & 0 & 0 & 0 & 1 & 1 & $C_{1}$ connected to $C$ \\
0 & 0 & 0 & 0 & 0 & 1 & 0 & 1 & $C_{2}$ connected to $C$ \\
0 & 0 & 0 & 0 & 1 & 0 & 0 & 1 & $C_{3}$ connected to $C$ \\
0 & 0 & 0 & 1 & 0 & 0 & 0 & 1 & $C_{4}$ connected to $C$ \\
0 & 0 & 1 & 0 & 0 & 0 & 0 & 1 & $C_{5}$ connected to $C$ \\
0 & 1 & 0 & 0 & 0 & 0 & 0 & 1 & $C_{6}$ connected to $C$ \\
\hline
\end{tabular}

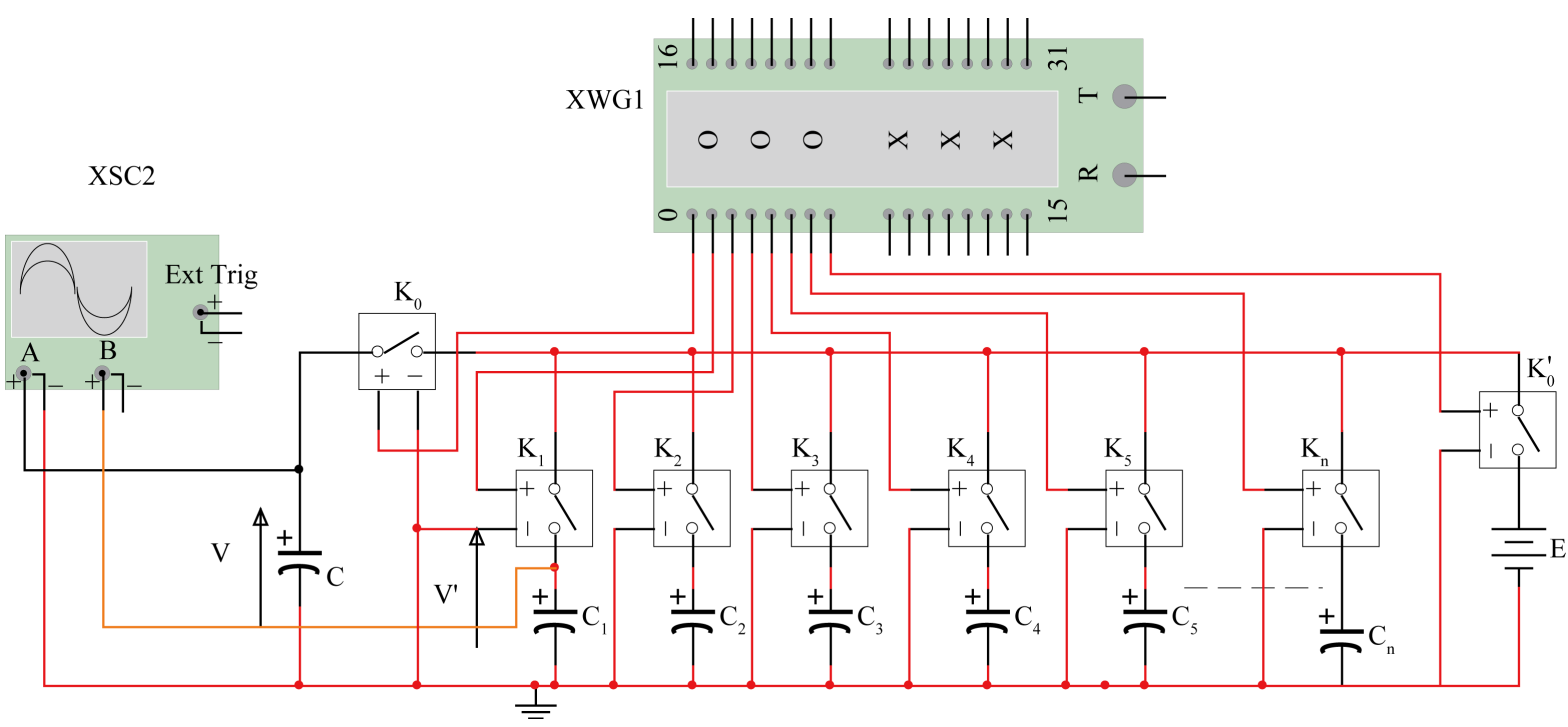

Figure 9. Diagram of different switching techniques.

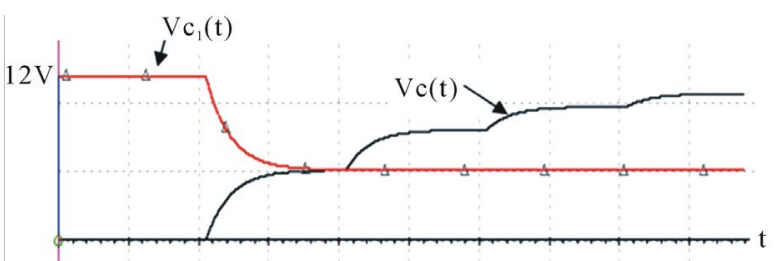

(a)

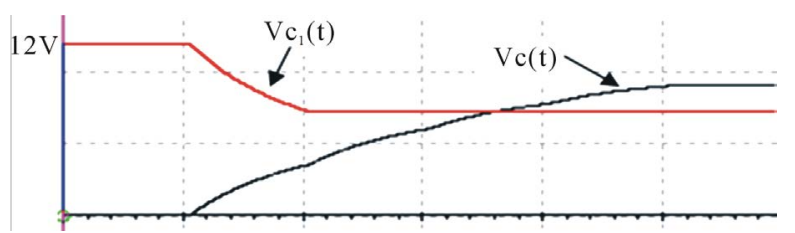

(b)

Figure 10. $V c=f(t)$ for $10 \mathrm{HZ}$ and $50 \mathrm{HZ}$. (a) $V c=f(t)$ (black) and $V c_{1}=f(t)$ (Red); (b) $V c=f(t)\left(\right.$ black), $V c_{1}=f(t)($ Red).

In Figure 11(b), we show a comparison of dynamic behavior of the charge $C_{1}$ in ICSP and in SCCSP. To improve the charge transfer process, we propose that, when any switched capacitor on $C$ is disconnected, it must be reconnected to the renewable source to be loaded again. The SC which will be connected to $C$ must be the one having the higher energy from the $C_{i}$; also we need to constantly know the status of the source and the state of charge (SOC) of each $C_{i}$, to be able to connect the intermittent source to the adequate capacitor $C_{i}$.

The following circuit diagram of Figure 12 allows us to conduct a synchronized time comparison between different switching techniques namely (Figure 13): SCCSP (Simple Cumulative Capacitor Switching Process), OCCSP (Optimal Capacitor Switching Process), SGSP (Simple Global Switching Process) and OGSP (Optimal Global Switching Process). 


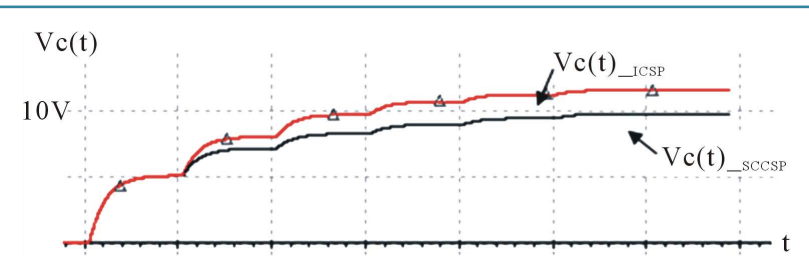

(a)

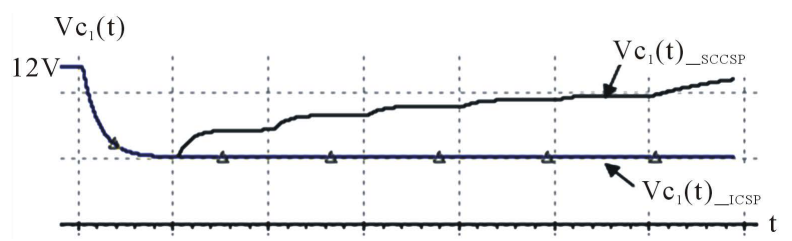

(b)

Figure 11. Dynamic behavior of $V_{C}=f(t)$ and $V c_{1}(t)$. (a) Dynamic behavior of $V_{C}=f(t)$ [ICSP (Red), SCCSP (Black)]; (b) Dynamic behavior of $V c_{1}(t)$ [ICSP (Blue), SCCSP (Black)].

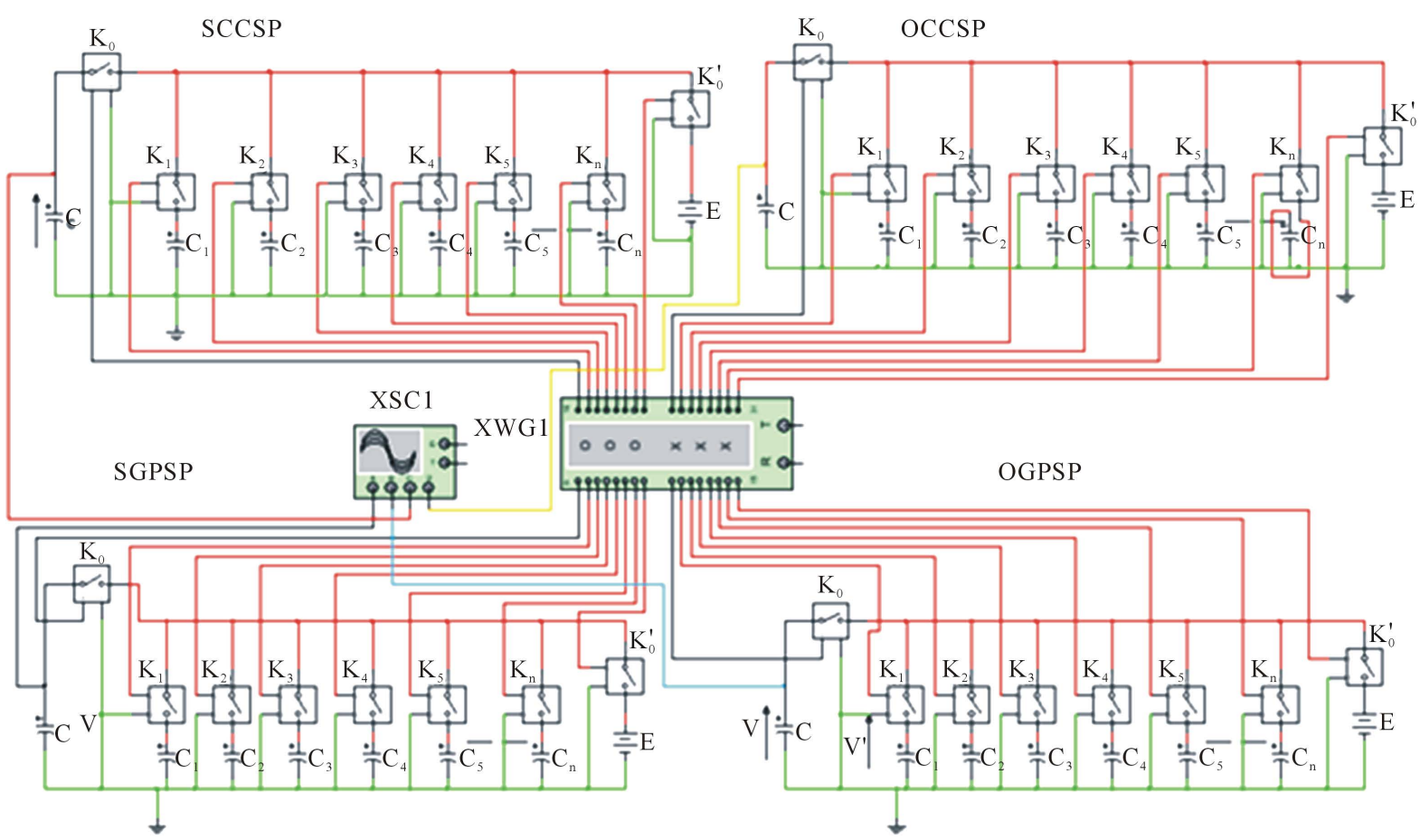

Figure 12. Diagram to conduct a synchronized time comparison between different switching techniques.

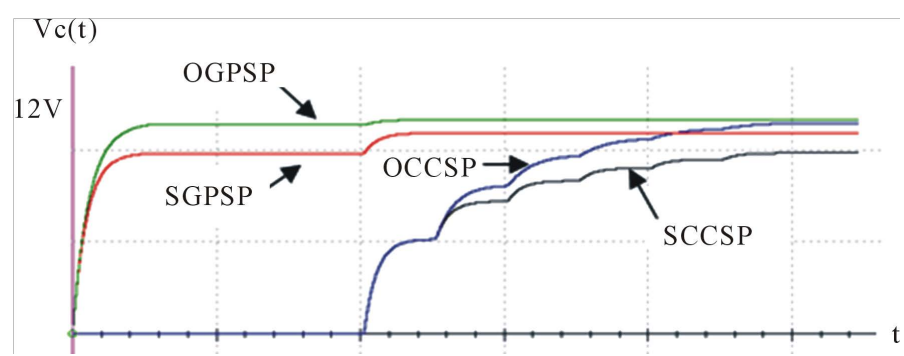

Figure 13. $V c=f(t)$ for different switching techniques.

We can easily improve the individual capacitor switching process. Indeed, all $C_{i}$ are first loaded to $E$, then connect $C_{1}$ to $C$, but let others $C_{i}$ connected to the source.

In the next step, isolate all $C_{i}$ from the source and disconnects $C_{1}$ from $C$ to be replaced by $C_{2}$.

At the same time, $C_{1}$ must be returned back to be reloaded from the renewable source. This process is applied for each of all the capacitors in turns.

\section{Conclusions}

In this paper, we present some technical methods to transfer electric charges from a renewable source to a sto- 
rage tank using a polymorphic capacitor circuit. This latter is mainly composed of SC and controlled switches; the system is controlled in real time, using an intelligent algorithm to transfer and store the maximum electric charge. The optimal solution for maximum energy transfer with minimum capacitors is the individual capacitor switching process (ICSP).

Research is still needed to better evaluate the potential of existing storage systems, develop new technologies and make them competitive in the new market environment.

Recently and in the future, polymorphic super-capacitors seem to achieve high performance thanks to their switching ability, long cycle life and their reliability to constitute independent means for energy storage.

\section{References}

[1] Raihani, A., et al. (2011) An Optimal Management System of a Wind Energy Supplier. Smart Grid and Renewable Energy, 2, 349-358. http://dx.doi.org/10.4236/sgre.2011.24040

[2] E. Journal (1998) Technologies for Tomorrow. IEEE Power Engineering Review, 18, 5-8.

[3] Directorate-General for Research Sustainable Energy Systems (2006) European Smart Grids Technology Platform. Smart Grids, Vision and Strategy for Europe's Electricity Networks of the Future. Technical Report.

[4] Glavin, M.E. and Hurley, W.G. (2012) Optimization of a Photovoltaic Battery Ultracapacitor Hybrid Energy Storage System. Solar Energy, 86, 3009-3020. http://dx.doi.org/10.1016/j.solener.2012.07.005

[5] Smith, T.A., Mars, J.P. and Turner, G.A. (2002) Using Super Capacitors to Improve Battery Performance. Proceedings of 33rd Power Electronics Specialists Conference, Cairns, 124-128.

[6] Kötz, R. and Carlen, M. (2000) Principles and Applications of Electrochemical Capacitors. Electrochimica Acta, 45, 2483-2498 http://dx.doi.org/10.1016/S0013-4686(00)00354-6

[7] Palma, L., Enjetti, P. and Howze, J.W. (2003) An Approach to Improve Battery Run-Time in Mobile Applications with Supercapacitors. Proceedings of Power Electronics Specialists Conference, Acapulco, 918-923.

[8] Cohen, M. and Smith, R. (2002) Here and Now: Ultra Capacitors Are a Standard Option. EVS19, Proceeding of the EVS19, 812-819.

[9] Chatzivasileiadi, A. et al. (2013) Characteristics of Electrical Energy Storage Technologies and Their Applications in Buildings. Renewable and Sustainable Energy Reviews, 25, 814-830 http://dx.doi.org/10.1016/j.rser.2013.05.023

[10] Alanen, R., Appetecchi, G., Conte, M., DeJaeger, E., Graditi, G., Jahren, S., et al. (2012) Electrical Energy Storage Technology Review. European Energy Research Alliance.

[11] Logerais, P.O., Riou, O., Camara, M.A. and Durastanti, J.F. (2013) Study of Photovoltaic Energy Storage by Supercapacitors through both Experimental and Modelling Approaches. Journal of Solar Energy, 2013, Article ID: 659014.

[12] Camara, M.A. (2011) Modelisation du stockage de l'energie photovoltaïque par supercondensateurs. Ph.D. Thesis, Université Paris-Est, Paris.

[13] Farzanehfard, H., Beyragh, D.S. and Adib, E. (2008) A Bidirectional Soft Switched Ultracapacitor Interface Circuit for Hybrid Electric Vehicles. Energy Conversion and Management, 49, 3578-3584. http://dx.doi.org/10.1016/j.enconman.2008.07.004

[14] Van Voorden, A., Ramirez-Elizondo, L., Paap, G., Verboomen, J. and van der Sluis, L. (2007) The Application of Super Capacitors to Relieve Battery Storage Systems in Autonomous Renewable Energy Systems. Proceedings of the IEEE Power Tech Conference, Lausanne, 1-5 July 2007, 479-484.

[15] Kuperman, A. and Aharon, I. (2011) Battery-Ultracapacitor Hybrids for Pulsed Current Loads: A Review. Renewable and Sustainable Energy Reviews, 15, 981-992. http://dx.doi.org/10.1016/j.rser.2010.11.010

[16] Henson, W. (2008) Optimal Battery/Ultracapacitor Storage Combination. Journal of Power Sources, 179, 417-423. http://dx.doi.org/10.1016/j.jpowsour.2007.12.083

[17] Dougal, R.A., Liu, S.Y. and White, R.E. (2002) Power and Life Extension of Battery-Ultracapacitor Hybrids. IEEE Transactions on Components and Packaging Technologies, 25, 120-131. http://dx.doi.org/10.1109/6144.991184

[18] Fang, X., Kutkut, N., Shen, J. and Batarseh, I. (2011) Analysis of Generalized Parallel-Series Ultracapacitor Shift Circuits for Energy Storage Systems. Renewable Energy, 36, 2599-2604. http://dx.doi.org/10.1016/j.renene.2010.05.003

[19] Dougal, R.A., Gao, L. and Liu, S. (2004) Ultracapacitor Model with Automatic Order Selection and Capacity Scaling for Dynamic System Simulation. Journal of Power Sources, 126, 250-257.

http://dx.doi.org/10.1016/j.jpowsour.2003.08.031

[20] Lu, R.G., Zhu, C.B., Tian, L.K. and Wang, Q. (2007) Super-Capacitor Stacks Management System with Dynamic 
Equalization Techniques. IEEE Transactions on Magnetics, 43, 254-258.

[21] Rebbani, A., Bouattane, O., Bahatti, L. and Zazoui, M. (2014) Efficient Charge Transfer Process in an Intelligent Electrical Storage System. IRACST-Engineering Science and Technology: An International Journal (ESTIJ), 4, 91-100.

\section{Notations}

SC: Supercapacitor.

ICSP: Individual Capacitor Switching Process.

CCSP: Cumulative Capacitor Switching Process.

SCCSP: Simple Cumulative Capacitor Switching Process.

OCCSP: Optimal Cumulative Capacitor Switching Process.

GPSP: Global Parallel Switching Process.

SGPSP: Simple Global Parallel Switching Process.

OGPSP: Optimal Global Parallel Switching. 
Scientific Research Publishing (SCIRP) is one of the largest Open Access journal publishers. It is currently publishing more than 200 open access, online, peer-reviewed journals covering a wide range of academic disciplines. SCIRP serves the worldwide academic communities and contributes to the progress and application of science with its publication.

Other selected journals from SCIRP are listed as below. Submit your manuscript to us via either submit@scirp.org or Online Submission Portal.
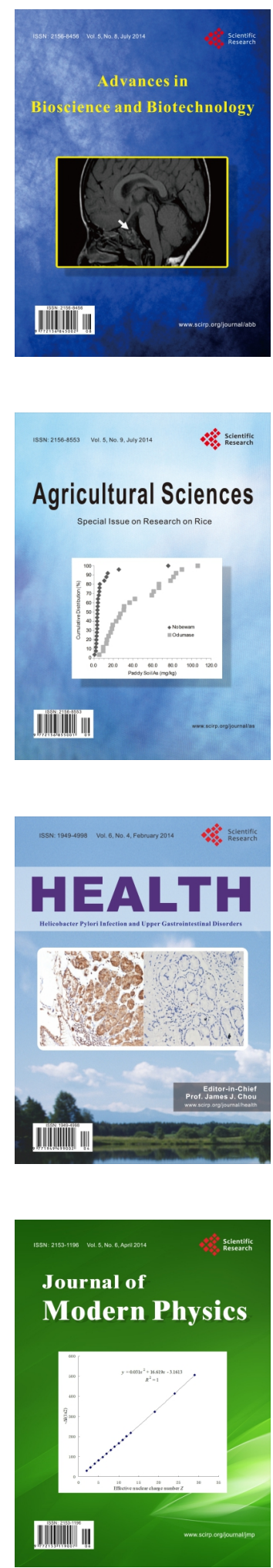
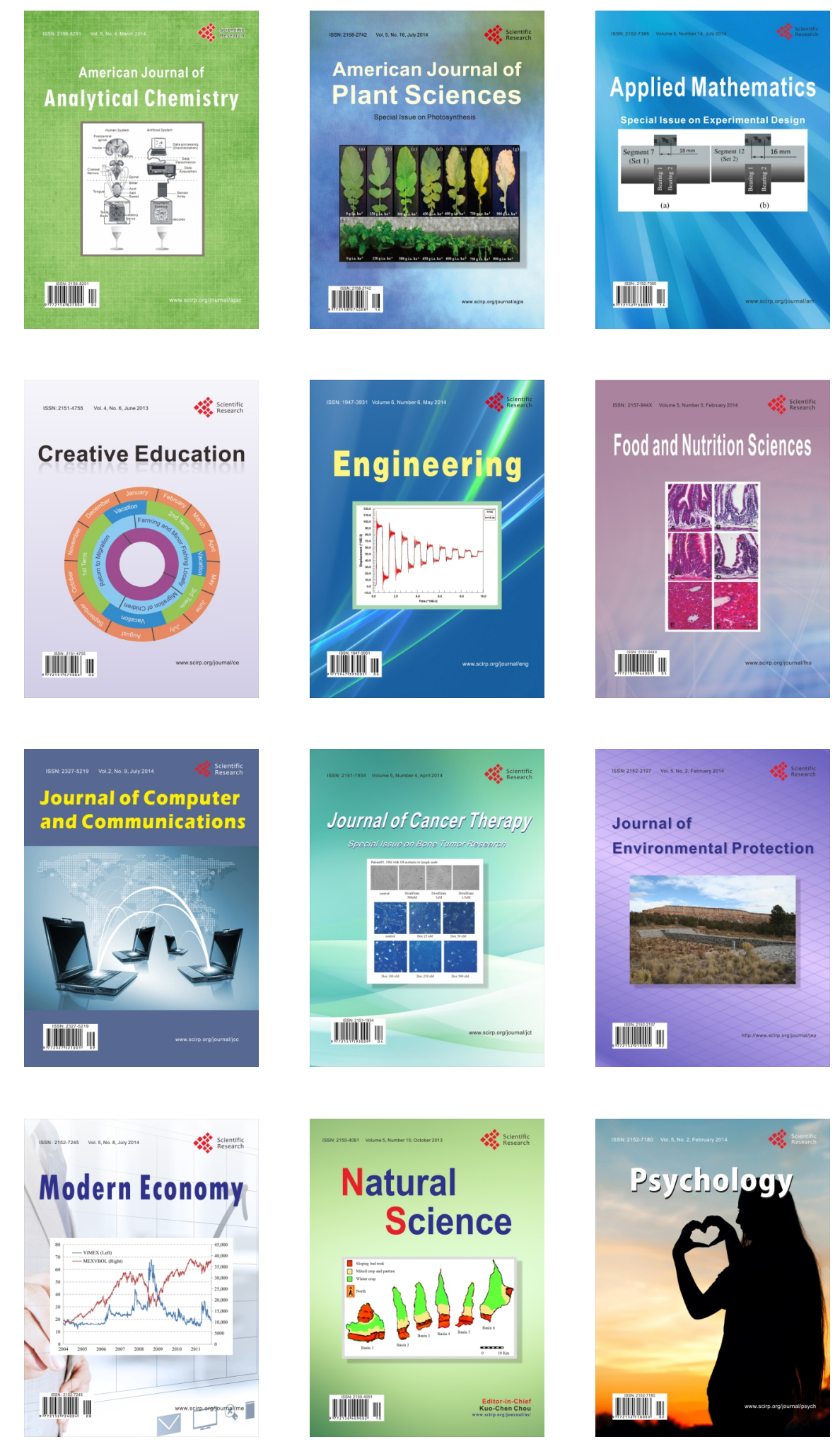